\title{
The Key Technologies about Energy Internet
}

\author{
Bin Li ${ }^{a}$, Shihui Yang ${ }^{b}$, Fangfang Duan, Kai Yan \\ State Grid Henan Electric Power Company Luoyang Power Supply Company, Luoyang 471900, \\ China \\ aluoyangdidiao@sina.com, b1015903240@qq.com
}

Keywords: Energy internet, smart grid, key technology.

\begin{abstract}
Energy internet provides a feasible technical solution to solve the problem of efficient use of renewable energy. Energy internet has certain unique in concepts, techniques, and methods with smart grid, distributed generation, micro grids. Therefore, the study of energy characteristics and implications of the Internet, explore key energy technology of the Internet, promoting the development of energy Internet, and gradually the traditional energy grid to the evolution of the Internet has important theoretical and practical value.
\end{abstract}

\section{Introduction}

Energy is the foundation of modern society's survival and development. In order to address the energy crisis, countries to actively research new energy technologies, especially solar, wind, biomass and other renewable energy sources. Renewable energy has an inexhaustible, clean and environmentally friendly features are highly valued around the world, scientists noted that the energy of the sun's rays irradiated one hour is sufficient to support the global economy throughout the year. The European photovoltaic Industry Association predicts that PV systems installed in all suitable building surfaces can generate 1.5 billion $\mathrm{kWh}$ of electricity to meet the required $40 \%$ of $\mathrm{EU}$ electricity.

Renewable energy exist geographically dispersed production is not continuous, centralized management approach randomness and uncontrollable volatility characteristics, the traditional power network, it is difficult to adapt to large-scale use of renewable energy requirement for effective renewable energy use is distributed "in situ collection, local storage, local use." but micro-grid and distributed generation grid does not fundamentally change the high penetration of distributed generation in the case of the primary electricity grid quality, fault detection, fault isolation effect, but also difficult to maximize the use of renewable energy sources, only renewable energy generation to share information in order to control the energy flow of information flow to achieve efficient transfer and sharing of renewable energy power generation capacity by in order to overcome the problems of instability of renewable energy, renewable energy utilization truly effective.

\section{The Features and Connotation of Energy Internet}

Energy Internet consists of several interconnected LANs energy constitutes Energy LAN router by the energy, power generation equipment, energy storage equipment, AC and DC load composition, and network can also be run independently off-grid Energy router from the solid state transformers (solid state transformer, SST) and intelligent energy management composition; intelligent energy management LAN based on the collected energy generation equipment, energy storage equipment and load control information to make energy decisions, then the control command to the solid state transformer execution, namely intelligent energy management control information flow, solid-state transformer control energy flow. To ensure reliable energy security of the Internet, the energy level of the bus LAN with intelligent fault management functions, providing real-time detection, rapid fault isolation functions Energy Internet Energy Internet and other forms of power compared to a system with the following four key features. 
Internet penetration in the high-energy. Renewable energy access a large number of various types of distributed renewable energy generation systems, renewable energy sources in the high permeability of environment, there is a big difference between control and management of the Internet with traditional energy grid needs research resulting series of new scientific and technical issues.

Nonlinear stochastic nature of distributed. renewable energy future is the subject of the Internet, but renewable energy has great uncertainty and uncontrollability, taking into account real-time pricing, changes in operating mode, the user-side response, load changes and other factors random nature, the Internet will show energy complex stochastic characteristics of the control, optimization and scheduling will face even greater challenges.

Multi-source big data characteristic. Energy internet in highly information-oriented environment, with distributed power grid, energy storage and demand response implementation, including meteorological information, users of electricity characteristics, energy storage state, and other sources vast amounts of information. Moreover, with the popularization and application of advanced metering technology, the Internet has the energy measurement function of the number of intelligent terminals will increase, it will dramatically increase the amount of data generated.

Multistage dynamic characteristic. Energy Internet is a matter, energy and information depth of the coupled system is a physical space, energy space, information space and the social space coupled multi-domain, associated multi-level, comprising a continuous dynamic behavior of discrete dynamic behavior and chaos conscious the behavior of complex systems. As a social / information / physical interdependence of large scale complex networks, compared with the traditional power grid, have a broader openness and greater system complexity, showing a complex and dynamic characteristics of the different scales.

\section{The Analysis of Key Technology about Energy Internet}

In order to solve the problems arising from the above features, the development of energy networks need to solve six key technologies: advanced energy storage technologies, solid-state transformer technology, intelligent energy management technology, intelligent fault management technology, reliable and secure communications technologies and systems planning analysis techniques.

\section{Advanced Energy Storage Technology.}

And the user-side nodes of different traditional grid, energy users on the Internet side node (such as a home or cell, etc.) generally have a power generation capacity, requiring with a certain scale distributed energy storage system. On the other hand the grid side energy Internet or power side, because of the high penetration of renewable energy sources, so in order to maintain stable operation of the system, must be equipped with large-scale centralized storage system can be seen, distributed and large-scale energy coexist is an important characteristic of Internet storage .

Distributed energy storage is mainly user-oriented, economic efficiency is crucial to the energy storage system storage efficiency, energy density, life and put forward higher requirements, new energy storage materials are key to improving the performance; the current large-scale storage main means battery group technology, energy storage unit after the battery into a group of scientific management is the storage system efficiency and long life operation important guarantee; whether the layout and construction of distributed energy storage or centralized storage, will on the whole energy Internet have a greater impact, so scientific and rational planning of the energy storage system is significant.

\section{Solid State Transformer Technology.}

With the high penetration of renewable energy generation equipment and energy storage devices to access traditional transformer power quality cannot meet the demand for energy and other aspects of the construction and development of the Internet, and solid-state transformer as a power electronic devices use high frequency energy and power control of converters, energy is considered to be the core technology of the Internet, the principle shown in Figure 3, the solid-state transformer consists of 
three parts, namely: AC / DC rectifier, DC / DC converters and DC / AC inverse variable is solid state transformer enables efficient management of renewable energy power generation and energy storage device and load solid state transformer has a two-way flow of energy capability, you can control the active and reactive power, with greater control bandwidth-play function.

\section{Smart Energy Management Technology.}

Energy Internet with a variety of energy generation equipment, power transmission equipment, energy consuming equipment, dynamic topology changes, with the typical characteristics of nonlinear stochastic dynamics and multi-scale features, in order to achieve energy LAN plug and play within energy devices "'Managing distributed more synergy between energy LAN control, and control strategies for renewable energy high robustness under high permeability need in energy layers of the Internet to introduce intelligent energy management technology.

\section{Intelligent Fault Management Technology.}

Intelligent fault management technologies in the energy Internet, the solid state transformer provides effective management of distributed energy and load, because of its strong limiting effect, can greatly improve the short-circuit current waveform, improve grid stability, compared with the traditional power grid, energy Internet fault current is very small, can only provide twice the rated current of the fault current, traditional fault detection apparatus and method to detect the current size of the failure, you need to design a new fault identification and location method. This requires the design of a new the circuit breaker, ensure that when the system fails, the breaker can quickly isolate the defective unit, making solid state transformer can quickly restore the system voltage. The traditional mechanical breaker causes the system in case of failure, the power flow brief the interruption will largely disturb the system's critical-load operation, while developed using solid-state power semiconductor devices instead of mechanical solid state crowbar circuit breakers to meet the energy needs of the Internet.

\section{Reliable and Secure Communication Technology.}

Secure and reliable communications backbone is an important guarantee for energy internet work properly because of internal energy LAN energy equipment are easy to contact with the high variability of dynamic topology, multi-scale dynamic energy of the Internet, making the energy complex Internet communication structure, data processing capacity; in order to ensure the stable operation of the energy of the Internet, a communications network to meet the requirements of network delay is small, the data transfer priority classification, reliable transmission, time synchronization, and support for multi-point transmission and other features.

Achieve a normal and efficient energy Internet communications network, we need to address the following issues: (1) devices that communicate variety (IEM, IFM, power generation equipment, smart load); (2) communication of different levels (WAN, regional networks, home area networks); (3) the communication delay require a higher $(<20 \mathrm{~ms})$.

\section{System Planning Analysis Technology.}

Energy Internet is a matter, energy and information depth coupled system, is the physical space, energy space, information space and the social space coupled multi-domain, associated multi-level, comprising a continuous dynamic behavior of discrete dynamic behavior and chaos conscious behavior of complex systems As social / information / physical interdependence of large scale complex networks, compared with the traditional power grid, have a broader openness and greater system complexity. energy internet system in the collaborative process between the nodes of control there by Game and a strong social impact of market price and changes in government policies and network topologies energy flow by, structure and unit heterogeneity, complex behavior, energy and information depth of integration, the amount of supply and demand characteristics of uncertainty, showing more mixed scale dynamic and complex network characteristics. Thus, the Internet system planning to conduct energy analysis technology research, analysis and control of the Internet reveals energy, operation and evolution mechanism of energy research in the Internet system design and optimization system, energy system planning and other aspects of the Internet basic theory and key technology is of great significance. 


\section{Summary}

Areas of the Internet involve a wide range of energy, such as materials science, biological science, control science, information science, management science, and economics, is a typical interdisciplinary problem. Energy efficient use of the Internet to solve the problem of renewable energy sources, provided feasible ideas and technical solutions This paper analyzes six key technologies in the energy of the Internet: advanced energy storage technologies, solid-state transformer technology, intelligent energy management technology, intelligent fault management technology, reliable and secure communications technologies, system planning analysis, a preliminary discussion each key technical scientific issues need to be addressed, the purpose is to draw attention to energy Internet research academia to jointly promote the development of the energy of the Internet, in order to solve the world's energy problems in China and contribute.

\section{Reference}

[1] Chenhang Yu, Ying Sun, Xiaona Niu, alt. Distributed renewable energy generation Internet system [J], Electric Power Automation Equipment,2010,5:104-108.

[2] Yabing Cha, Tao Zhang, ShurenTan.alt. Understanding and Thinking on Energy Internet [J] National Defense Science and Technology, 2012, 5:1-6.

[3] Bo Zhao, Peng Li, Hangwei Tong.alt. Summary of Research of Distributed Generation to Micro Grid[J]. Zhejiang Electric Power.2010.3:1-5

[4] ShaoYong Wang, Micro-based distributed power network design and operation [J] Electric Power Automation Equipment.2011, 4:120-123.

[5] Junwei Cao, Kun Meng, JiyeWang.alt. Energy Internet router and Energy [J] Chinese Science: Information Science, 2014, 44 (6): 714-727.

[6] Zhaoyang Dong, Junhua Zhao, Shuan Fu.alt. From Smart Grid to Energy Internet:Basic Concept and Research Framework[J].2014,15:112-150 